\title{
Review
}

Journal of Innate

Immunity
$\mathrm{J}$ Innate Immun 2010;2:204-215

DOI: $\underline{10.1159 / 000296507}$
Received: December 21, 2009

Accepted after revision: February 10, 2010

Published online: March 16, 2010

\section{Monocytes and Macrophages Regulate Immunity through Dynamic Networks of Survival and Cell Death}

\author{
Arti Pariharc Timothy D. Eubank $^{a} \quad$ Andrea I. Doseffa, ${ }^{a}$ \\ ${ }^{a}$ Department of Internal Medicine, Division of Pulmonary and Critical Care, Heart and Lung Research Institute, and \\ ${ }^{b}$ Department of Molecular Genetics, The Ohio State University, Columbus, Ohio, USA; ' Department of Biological \\ Sciences, GDC College, Vikram University, Ujjain, India
}

\section{Key Words}

Apoptosis $\cdot$ Cell fate $\cdot$ Macrophages $\cdot$ Monocytes .

Signal transduction proteins

\begin{abstract}
Monocytes and macrophages are central cells of the innate immune system, responsible for defending against diverse pathogens. While they originate from a common myeloid precursor and share functions in innate immunity, each has a very distinct life span finely tuned by the apoptotic caspases. Normally, circulating monocytes are short-lived and undergo spontaneous apoptosis on a daily basis. Macrophages, however, have a longer life span. In chronic inflammatory diseases and, as recently recognized, in the tumor microenvironment, the inhibition of the apoptotic program promotes monocyte survival contributing to the accumulation of macrophages and the persistence of an inflammatory milieu. A complex network of differentiation factors and inflammatory stimuli determine monocyte/macrophage life span by blocking the apoptotic pathway and activating a myriad of survival pathways. Our understanding of apoptosis has flourished over the last decade, and its relevance in the regulation of the immune system is now indisputable. Nevertheless, how the complicated networks of survival and apoptotic regulators are integrated to determine cellular life
\end{abstract}

span remains elusive. This review summarizes the contribution of the caspases and their regulators in monocyte/macrophage cell fate and discusses how these molecules orchestrate the initiation, maintenance, and resolution of inflammation. More provocatively, we discuss possible strategies to control inflammation by manipulating leukocyte life span.

Copyright $\odot 2010$ S. Karger AG, Basel

\section{Introduction}

Monocytes and macrophages are essential components of the innate immune system. They comprise what was just recently recognized as a heterogenous family of professional phagocytic cells responsible for the recognition and clearance of pathogens and dead cells. Monocytes and macrophages play central roles in the initiation and resolution of inflammation, principally through phagocytosis, release of inflammatory cytokines, reactive oxygen species (ROS) and the activation of the acquired immune system [1]. Monocytes and macrophages originate from a common myeloid progenitor cell in the bone marrow. Under normal circumstances, monocytes circulate in the bloodstream for a very short time before undergoing spontaneous apoptosis [2]. In response to differentiation factors, monocytes escape their apoptotic

\section{KARGER}

๑๐ 2010 S. Karger AG, Basel

Fax +4161306 1234

E-Mail karger@karger.ch

www.karger.com
Accessible online at:

www.karger.com/jin
Dr. Andrea I. Doseff

Heart and Lung Research Institute, The Ohio State University 473 West 12 th Ave.

Columbus, OH 43220 (USA)

Tel. +1 614292 9507, Fax +1 614293 4799, E-Mail doseff.1@ osu.edu 
fate by differentiating into macrophages, cells with a longer life span found in almost every single organ [3]. A balanced network of survival and cell death proteins determines the fate of these cells. For example, increased levels of the small heat shock protein 27 (Hsp27) in macrophages inhibit the activation of caspase-3, an essential caspase for monocyte apoptosis [4]. The presence of stimulatory signals triggers monocyte survival by inhibiting the apoptotic pathway, thus contributing to the maintenance of the inflammatory response [5]. Then, as inflammation resolves, almost like under a 'magical spell', the apoptotic program reassumes and monocytes undergo apoptosis, facilitating the resolution of the immune response [6].

Although monocytes represent an important part of the host defense, accumulation of monocytes can be harmful and aggravate diseases such as atherosclerosis, arthritis and multiple sclerosis [7]. Recently, the realization that monocytes/macrophages play fundamental biological roles in development, wound healing, tissue homeostasis and even cancer progression prompted an urgency to understand the molecular mechanisms that determine their life span and cell fate. Considering the plasticity of monocytes/macrophages to change their life span depending on the external and internal cellular cues, it is likely that complex survival and apoptotic molecular networks are in place to orchestrate cell fate. A better understanding of how to integrate these networks should provide novel approaches on how to regulate monocyte/macrophage accumulation at sites of inflammation. In this review we critically evaluate various biological aspects and processes of monocyte/macrophage survival and death signaling.

\section{Monocytes: Emerging Roles of Monocyte Subpopulations}

Monocyte heterogeneity is well accepted and their contribution to disease progression seems to be clearly diverse. Broadly, these subsets are divided into 'classical' and 'non-classical' on the basis of differential expression of antigenic markers and emerging recognized biological responses. The majority of monocytes (approx. 90-95\%) are defined as classical and strongly positive for surface receptor CD14 [lipopolysaccharide (LPS)-binding protein receptor CD14/Toll-like receptor TLR-4/MD2] and CD16 (Fc $\gamma$ RIII)-negative [8]. The classical population expresses Fc $\gamma$ RI (CD64). The non-classical subpopulation is characterized by the expression of $\mathrm{CD}^{2} 6^{+}$. The non- classical $\mathrm{CD} 14^{+} \mathrm{CD} 16^{+}$can be further divided into 2 other subpopulations, namely $\mathrm{CD} 14^{\text {bright }} \mathrm{CD} 16^{+}$and $\mathrm{CD} 14^{\mathrm{dim}} \mathrm{CD} 16^{+}$monocytes, with strikingly different functions $[9,10]$. Pioneering work by Ziegler-Heitbrock [11] and others uncovered the crucial role of $\mathrm{CD} 14^{+} \mathrm{CD} 16^{+}$ in inflammation. Upon stimulation, $\mathrm{CD} 14^{+} \mathrm{CD} 16^{+}$are capable of producing TNF $\alpha$ and IL- $1 \beta$. But the $\mathrm{CD} 16^{+}$subgroups differ in their ability to release IL-10. In contrast to $\mathrm{CD} 14^{\text {bright }} \mathrm{CD} 16^{+}, \mathrm{CD} 14^{\mathrm{dim}} \mathrm{CD} 16^{+}$and classical $\mathrm{CD} 14^{+} \mathrm{CD} 16^{-}$produce very low amounts of IL-10 when stimulated with LPS [10]. CD14 ${ }^{\text {bright }} \mathrm{CD} 16^{+}$monocytes also have high expression of CD11b and TLR4 in comparison to $\mathrm{CD} 14^{+} \mathrm{CD} 16^{-}$and $\mathrm{CD} 14^{\mathrm{dim}} \mathrm{CD} 16^{+}$. In addition, $\mathrm{CD} 14^{\text {bright }} \mathrm{CD} 16^{+}$and $\mathrm{CD} 14^{\mathrm{dim}} \mathrm{CD} 16^{+}$express high levels of HLA-DR, CD86, CD54 and low levels of CD64 in comparison to the major classical subpopulation of monocytes. The actual function of the $\mathrm{CD} 14^{+} \mathrm{CD} 16^{+}$monocytes remains elusive, but these cells may be expanded in the blood of patients with conditions such as sepsis, pulmonary tuberculosis, AIDS and cancer. It may therefore be an oversimplification to state unambiguously that the $\mathrm{CD} 14^{+} \mathrm{CD} 16^{+}$monocytes act as pro-inflammatory monocytes during all in vivo infections. In this regard, interesting studies showed increased $\mathrm{CD} 14^{+} \mathrm{CD} 16^{+}$numbers mobilized from the marginal pool during exercise, suggesting a dynamic localization of specific subpopulations [11]. Further studies have shown that monocyte subpopulations also differ on other surface molecules, such as the chemokine receptors CCR2, CCR5 and CX3CR1. CCR5 and CX3CR1 are highly expressed on the minor subset $\mathrm{CD} 14^{\mathrm{dim}} \mathrm{CD} 16^{+}$, while the expression of CCR 2 and lower levels of CX3CR1 are characteristic of the CD14 ${ }^{\text {bright }}$ $\mathrm{CD}^{-} 6^{-}$monocytes [12]. The expression of specific chemokine receptors and adhesion molecules might have important functional consequences for these cells, resulting not only in differences in migration patterns but also conferring differential susceptibility to infections [13]. Increased numbers of $\mathrm{CD} 14^{\mathrm{dim}} \mathrm{CD} 16^{+}$have been reported during infections in humans, including hemolytic uremic syndrome, bacterial sepsis and HIV, and also in experimental SIV infections in monkeys. Great progress during the recent years using animal models has provided a better understanding of the complex monocyte heterogeneity, their origins and localization [for an extensive review see 14]. Notably, recent comprehensive studies comparing human and mice monocyte gene expression profiles showed the expected conservation between similar monocyte subsets in mice and humans; however, some important differences found between the species highlight the limitation of using animal models to under- 
stand human diseases [15]. Future studies comparing the protein expression profile will also be of value to better characterize specific signatures of each subpopulation.

The emerging realization is that these subsets have different behaviors contributing to many diseases. As better reagents and models emerge, the possibility to dissect these subsets and determine their individual contributions becomes vital for the implementation of novel treatments based on modulating monocyte numbers, function and life span.

\section{Heterogenous Macrophages}

Macrophages are large phagocytic cells originally identified by Aschoff [16]. They are found in almost every organ and were historically given different names based on their tissue in which they reside. Due to great advances in the study of the tumor microenvironment in the last decade, it has become even more obvious that macrophages constitute a highly heterogeneous pool with distinct biological activities that are influenced both by genetics and environment.

Macrophages originate from monocytes in response to differentiation factors such as granulocyte-macrophage colony-stimulating factor (GM-CSF), macrophage colony-stimulating factor (M-CSF), and colony-stimulating factor-1 (CSF-1). GM-CSF-deficient mice have normal hematocrit and leukocyte numbers but develop lymphoid hyperplasia in the lungs [17]. M-CSF-deficient mice show extensive skeletal deformities and reduced numbers of blood monocytes, peritoneal macrophages and tissue macrophages, suggesting its fundamental role in monocyte and macrophage biogenesis [18]. Comparative studies have demonstrated differences in gene expression between monocytes and macrophages and their different life spans. Unlike monocytes, macrophages have a long life span, ranging from months to years [19]. Changes in transcription factors and surface receptor expression showed that macrophages express decreased CD14 and increased levels of CD11b, mannose receptor [MR or CD206], the transcription factor PU.1, surface receptors MHC-II, Fc $\gamma \mathrm{R}$ and scavenger receptor, among others [20]. In addition, differences in the expression of molecules involved in survival and cell death have been documented. Stimulation of monocytes with phorbol ester 12-O-tetradecanoylphorbol-13-acetate shows a dramatic increase of the anti-apoptotic Hsp27, and decreased expression of the pro-apoptotic protein kinase $\mathrm{C} \delta$ (PKC $\delta$ ) [21]. In agreement with these results, we found a 40-fold increase in the expression of Hsp27 protein during monocyte/macrophage differentiation [4]. Similarly, comparative proteomic analysis of monocytes and alveolar macrophages showed the specialized function of alveolar macrophages in phagocytic functions.

A tumor represents a fantastic model system to study monocyte/macrophage biology. In fact, it has been shown that increased recruitment of macrophages to solid tumors leads to both poor prognosis and outcome. Interestingly, it has been demonstrated that individuals taking nonsteroidal anti-inflammatory drugs (NSAIDs) have a reduction in the risk of developing certain cancers [22]. Canonically, both monocytes and tissue macrophages are recruited to the site of a tumor to initiate an anti-tumor response. Two subtypes of macrophages found in tumors are 'M1' and 'M2' macrophages. M1 macrophages are termed for their ability to assist in a T helper-1 (Th1) immune response mediated by IFN- $\gamma$, IL- $1 \beta$ and TNF $\alpha$. M1 macrophages present antigens to B cells, express IL-12, IL-23 and other inflammatory cytokines, and produce reactive oxygen and nitrogen species, which aid in their tumor-fighting function [23]. On the contrary, M2 macrophages or 'tumor-associated macrophages' (TAMs) are derived from direct tumor cell interaction and exposure to IL-4, IL-10, IL-13 and glucocorticoid hormones and instigate a Th2 response which acts to both up-regulate the expression of anti-inflammatory cytokines and down-regulate the production of pro-inflammatory mediators. M2 cells are characterized by IL-4 production, high expression of IL-10 and TGF $\beta$, release of immune complexes, and their ability to promote angiogenesis by expressing VEGF, and tissue remodeling and repair by expressing matrix metalloproteinases [23, 24]. TAMs are critical in tumor progression and are represented by a myriad of macrophage subtypes that do not resemble classical M1 macrophages that express the anti-tumor program described above.

Interestingly, macrophage subtypes may not be predetermined prior to recruitment but 'programmed' or 'educated' by the tumor microenvironment upon arrival. If so, the potential to manipulate and 're-educate' these TAMs back to an M1 phenotype can provide a novel opportunity to alleviate diseases characterized by monocyte/macrophage infiltration. In fact, recent advances by Eubank et al. [25] suggest an effectiveness of GM-CSF to help inhibit the growth of both murine breast cancer in an orthotopic PyMT model and in human breast cancer in SCID mice [unpubl. data]. It is reported that superphysiological doses of GM-CSF administered in the local tumor environment induces the production of sVEGFR-1 
from macrophages, resulting in the suppression of tumor-produced VEGF and angiogenesis [26]. Fortuitously, GM-CSF recruits significantly more macrophages to these tumors than those untreated. At one time, this effect might be considered a detriment to tumor therapy as a number of studies have shown that removal of TAMs leads to a reduction in angiogenesis and metastases [24]. But, because GM-CSF seems to either maintain the M1 macrophage phenotype recruited into the tumor, or because GM-CSF 're-educates' or re-programs' the M2 TAMs back to an M1 phenotype, this suggests that an increase in M1 macrophages is beneficial and illustrates that influencing the M1/M2 polarity of macrophages provides an opportunity for the host immune cells to once again perform their initial role as tumor-fighting cells.

Recent studies have shown that a unique subpopulation of monocytes, namely $\mathrm{CD} 14^{\mathrm{dim}} \mathrm{CD} 16^{+} \mathrm{CCR} 2^{-}$which makes up about $20 \%$ of $\mathrm{CD} 14^{+} \mathrm{CD} 16^{+}$cells, expresses the endothelial cell marker Tie-2, which falls into the category of host immune cells that aid tumor growth and angiogenesis. Tie-2 is a receptor for angiopoietin-2 (Ang-2), an angiogenic growth factor produced by blood vessels under hypoxia to recruit Tie-2-expressing endothelial cells. Consequently, a population of Tie-2-expressing monocytes is also recruited, which maintain their monocyte morphology subsequent to extravasation and diapedesis, and reside in perivascular regions to promote angiogenesis. Depletion of Tie-2-expressing monocytes has been shown to suppress tumor progression and metastases [27].

Studies such as this suggest it may be advantageous to focus on other monocyte/macrophage populations which are selected for in both peripheral blood and tissue of patients with diseases. The mechanisms of recruitment into tissues seem to differ between classical and non-classical monocytes. In particular, the expression levels of $\mathrm{L}$-selectin (CD62-L), a molecule central in mediating the adhesion of monocytes to the endothelium and their extravasation into the intima during the key steps in atherogenesis, is lower in $\mathrm{CD}^{+} 6^{+}$compared to classical $\mathrm{CD} 14^{+} \mathrm{CD} 16^{-}$. A dramatic expansion of $\mathrm{CD}^{+} 6^{+}$monocytes has been described in atherosclerosis patients, and this population showed increased L-selectin expression compared with the levels found in $\mathrm{CD}^{+} 6^{+}$from normal individuals. Additionally, asthma patients have increased numbers of $\mathrm{CD} 14^{\mathrm{dim}} \mathrm{CD} 16^{+}$monocytes expressing the hemoglobin scavenger receptor $\mathrm{CD} 163$, which plays a role in an anti-inflammatory response. As discrepancies based on technical differences arose, the contribution of the CD163 receptor remains controversial, and further investigations will help to establishing its contribution (see table 1 for more diseases and corresponding cell subpopulations).

The aforementioned studies highlight the potential for novel therapeutic applications based on the manipulation of each unique disease microenvironment. It is now becoming likely that specific monocyte/macrophage subpopulations are representative of certain diseases that involve the regulation and maintenance of unique subtypes of immune cells. Certainly, once in the tissue microenvironment, effects such as cytokine expression profile from surrounding cells as well as oxygen tension play a role in which cells progress the disease and which cells fight the disease. In such cases, is it possible that treatment strategies involving manipulation of proliferating immune cell subtypes in systemic blood may help control the inflammatory regulation that leads to the pathology of a disease? Looking ahead, improvements in microdissection techniques coupled with new immunolabeled antibodies should allow both a better characterization of these cells and superior evaluation of such treatments.

\section{Monocytes/Macrophages and Their Role in Innate Immunity}

Monocyte/macrophage surveillance is essential in the initial host reaction to infection by initiating an inflammatory response. The activation of monocytes/macrophages is triggered by the recognition of self and non-self stimuli mediated through a myriad of specialized membrane and intracellular receptors. The MHC (major histocomaptibilty complex) receptor family is classified as class I and II, based on the structure of their extracellular chains, also referred to as C1a or HLA-DR and CD38 or HLA-DQ, respectively. Monocytes express MHC-I, but during differentiation MHC-I expression is inhibited while MHC-II is induced [28]. The Toll-like receptor (TLR) family constitutes an evolutionarily conserved 'pathogen-recognition-receptors' protein group that recognizes conserved 'pathogen-associated-microbial-patterns' composed of 9 members involved in pathogen recognition. TLRs are type I integral membrane glycoproteins, containing an extracellular domain with leucine-rich repeat motifs and a cytoplasmic domain homologous to IL-1R (TLR) responsible for 'self'-signal recognition (fig. 1). In monocytes and macrophages TLRs 1, 2, 4, 5,6 and 10 have been identified in the plasma membrane. Intracellularly, TLRs 3, 7 and 9 are found in the endosome 
Table 1. Mononuclear phagocyte subpopulations in human disease

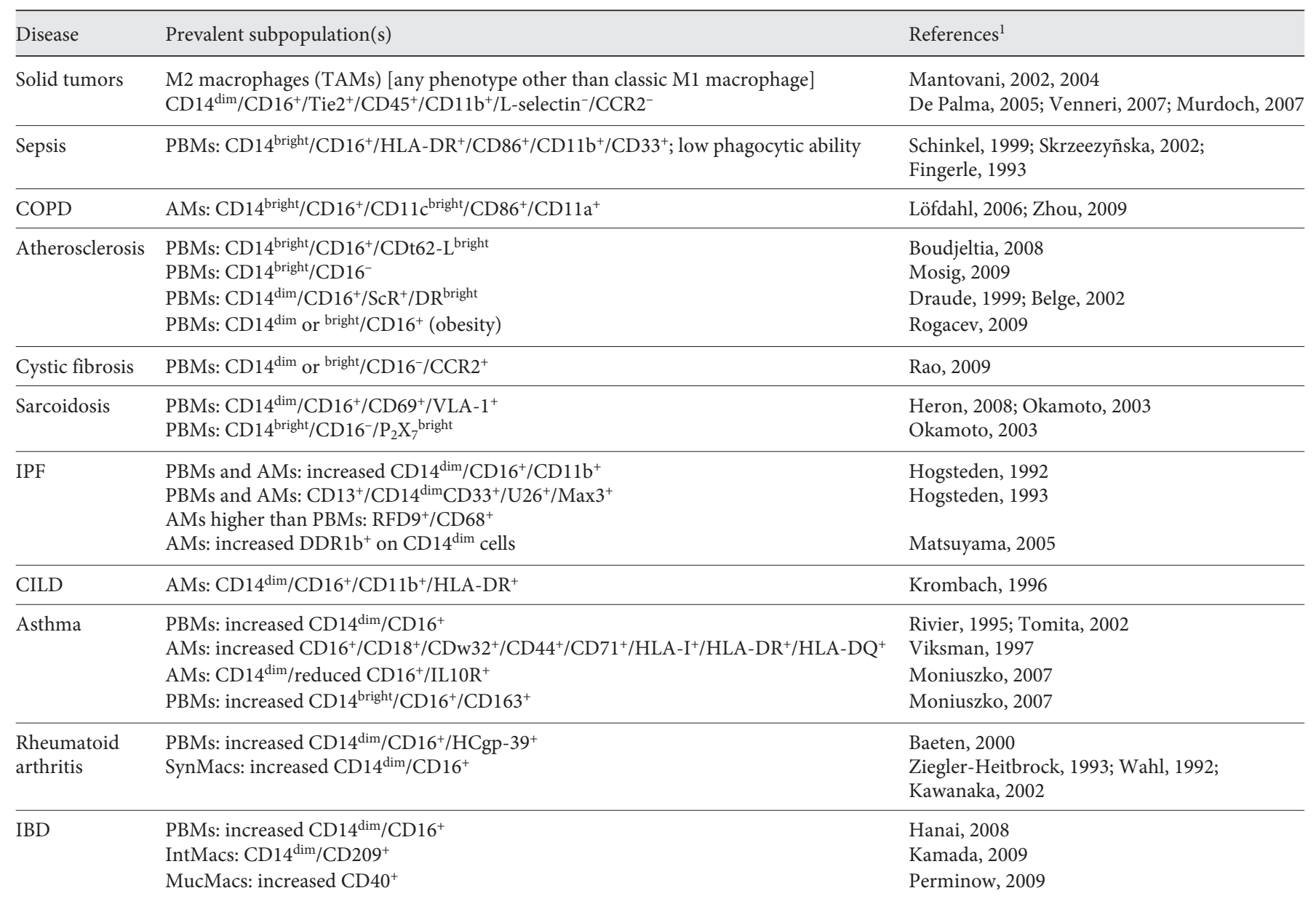

Receptor expression: 'dim' = +; 'bright' = ++. AMs = Alveolar macrophages; PBMs = peripheral blood monocytes; COPD = chronic obstructive pulmonary disorder; $\mathrm{IPF}=$ idiopathic pulmonary fibrosis; $\mathrm{CILD}=$ chronic inflammatory lung disease; $\mathrm{IBD}=$ inflammatory bowel disease; $\mathrm{TAMs}=$ tumorassociated macrophages; SynMacs = synovial fluid macrophages; IntMacs = intestinal lumen macrophages; MucMacs = mucosal macrophages.

${ }^{1}$ Only first author is shown (for full references see www.karger.com/doi/10.1159/000296507.

membranes, whereas only TLR9 is found in the endosome membranes in monocytes. TLR4 recognizes LPS, the major constituent of the outer wall of Gram-negative bacteria and one of the most studied activators of monocytes/macrophages [29]. Activation of TLR4 is mediated by a signal transduction pathway involving the myeloid differentiation factor 88 (MyD88) inducing the formation of the TRAF6/IRAK/MyD88 complex mediated by the activation of IRAK (IL-1R-associated kinases). Four IRAKs have been identified but only IRAK1 and IRAK4 show kinase activity and are central for the activation of NF-кB. IRAK4 is required for TLR signaling, as IRAK4deficient mice showed no response to IL- $1 \beta$ or LPS, and patients with inherited IRAK4 mutations fail to respond to IL-1 $\beta$ [30]. In addition, IRAK1-deficient mice showed decreased cytokine production in response to LPS, suggesting a sufficient, yet non-essential, role for the proper production of inflammatory cytokines. Activation of IRAK1/4 induces the recruitment of the adaptor molecules MyD88 and TRAF6 (TNF receptor-associated factor 6), an E3 ubiquitin ligase, resulting in the formation of the MyD88/IRAK/TRAF6 complex. Formation of this complex promotes the activation of a kinase cascade that involves the MAPK (mitogen-activated protein kinases), TAK1 (TGF- $\beta$-activated kinase 1 ) and/or MEKK3. The activation of these kinases leads to the activation of the 


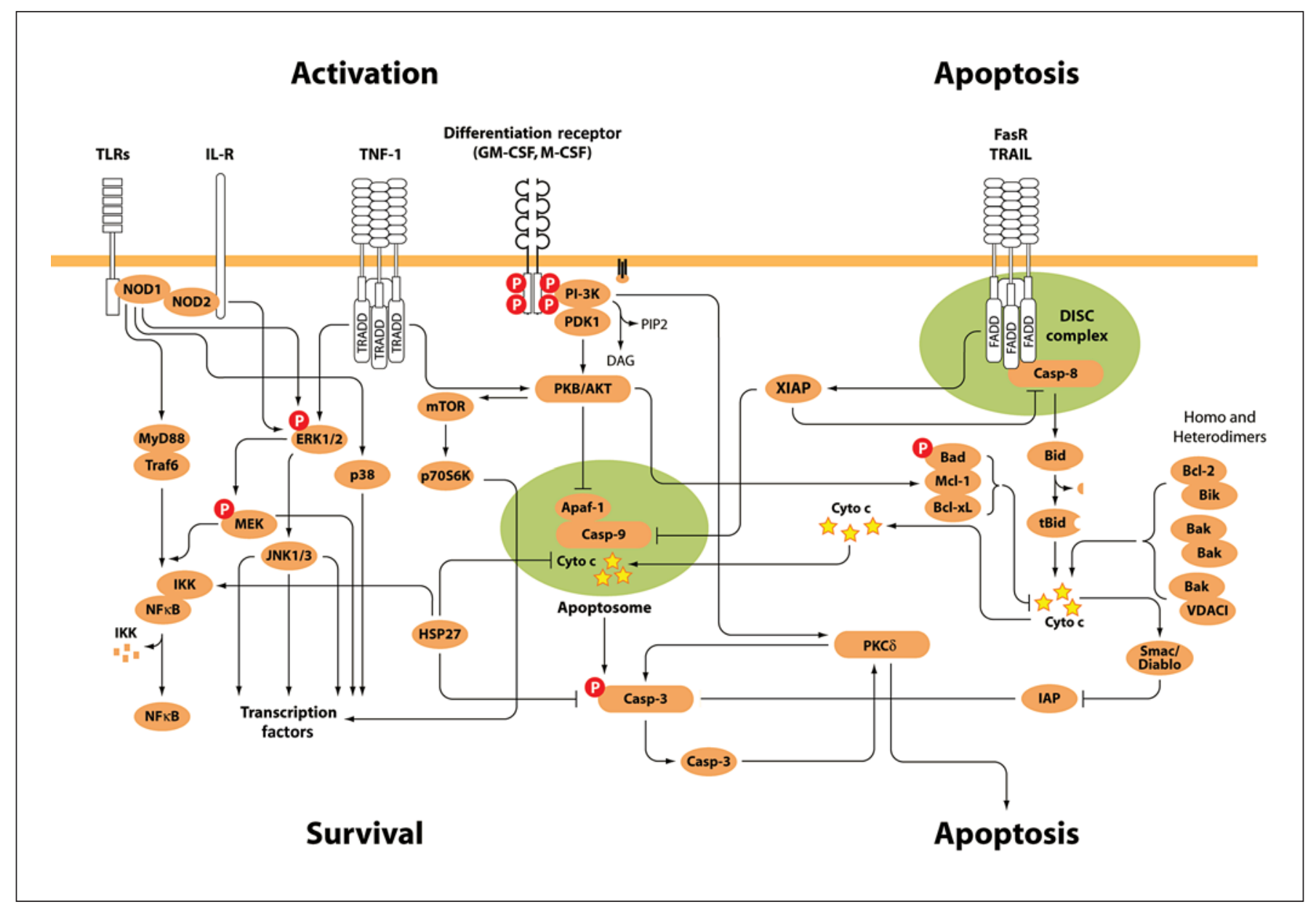

Fig. 1. Signaling networks regulating monocyte and macrophage life span.

IKK complex responsible for controlling NF- $\kappa$ B. The IKK complex is formed by 2 kinase-containing subunits, IKK $\alpha$ and $\beta$, and a regulatory subunit IKK $\gamma$ phosphorylates NF- $\kappa \mathrm{B}$ inducing NF- $\kappa \mathrm{B}$ transcriptional activity [31] leading to the production of pro-inflammatory cytokines and chemokines. Stimulation of TLRs typically induces the activation of the MAPKs p38, extracelluar signal-regulated kinase, and c-jun-terminal kinase (JNK) by pathways that remain to be fully characterized. Altogether, the stimulation of TLRs leads to the production of proinflammatory mediators such as IL- $1 \beta$ and TNF $\alpha$, among others. These cytokines are responsible for mediating immunity and inducing prolonged monocyte survival [5]. In some cases, TNF $\alpha$-induced apoptosis of alveolar macrophages has been reported in Mycobacterium tuberculosis infection. In this regard, conflicting data regarding the effect of cytokine survival stimuli in apoptosis might be due to differences on dosage or length of the treatment. In addition, these effects may just reflect the unique regulation of the survival/apoptotic networks in heterogeneous populations of monocytes and macrophages [8]. Notably, recognition of a 'self'-stimulatory signal such as IL-1 shares great similarities with 'non-self recognition' in terms of receptor and signal transduction conservation. In addition, monocyte activation can also be attained through the interaction between monocytic CD 40 with the CD40 ligand present on activated lymphocytes, leading also to prolonged monocyte survival. Further, monocyte activation can be mediated by their interaction with platelets, in parallel with the increased surface expression of Mac-1 (CD11b/CD18) and proteolytic shedding of L-selectin, release of superoxide anion, and increased tissue factor expression [32]. 
In inflammatory diseases such as atherosclerosis, monocytes are activated and recruited to the developing lesion of the arterial wall. Increased production of monocyte chemoattractant protein-1 (MCP-1/CCL2) and ROS is characteristic of the activation process. As a result, monocytes increase the production of cytokines such as IL-8, IL-1 $\beta$, and TNF $\alpha$ that further contribute to the local inflammation. An excessive production of these pro-inflammatory mediators has been associated with multiple organ system failure [33]. Once inflammation is established in the affected tissue, the second phase described for the classical monocytes is the production and release of anti-inflammatory mediators such as TGF- $\beta$, IL-10, IL-13, IL-4 and prostaglandin E2 in an effort to counteract ongoing inflammation.

Monocyte activation confers survival signals essential for the functional integrity of monocytes. This enables cells to remain viable in microenvironments of immune or inflammatory lesions that are rich in cytotoxic inflammatory mediators and reactive free radical species. However, prolonged activation can be deleterious and has been implicated in the pathogenesis of many inflammatory diseases including atherosclerosis, rheumatoid arthritis and tumor development. As a consequence of these findings, therapeutic approaches to target inflammation are based on the ability to reduce inflammatory cytokines. Anti-TNF $\alpha$ therapies have been used in patients with inflammatory conditions, such as rheumatoid arthritis and chronic colitis. However, these studies have reported only a $50-60 \%$ success rate. In support of these results, it could be of great significance to define alternative therapeutic approaches targeting activated monocytes to undergo apoptosis. This approach could help reduce inflammatory cytokines and at the same time contribute to the clearance of activated monocytes at sites of inflammation.

In fact, the potential use of plant flavonoids as antiinflammatory nutraceuticals is emerging as a potential alternative therapeutic approach targeting both pro-inflammatory mediators and monocyte numbers. Flavonoids are known for their anti-inflammatory, anti-oxidant, anti-viral, anti-microbial, and anti-allergic antiproliferative, and anti-metastatic properties. Flavonoids can scavenge ROS, chelate iron ions and inhibit lipid oxidation. Curcumin, obtained from rhizome of Curcuma longa and abundant in Asian diets, has strong anti-inflammatory and anti-oxidant properties inhibiting proinflammatory mediators such as TNF $\alpha$ and COX-2 by modulating NF- $\kappa \mathrm{B}$ [34]. Apigenin, a flavone abundant in the Mediterranean diet, has potent anti-inflammatory activity. We showed that apigenin inhibits the production of pro-inflammatory cytokines in LPS-stimulated human monocytes and mouse macrophages by negatively modulating the phosphorylation of NF- $\mathrm{BB}$ [35]. In fact, apigenin is more effective as an anti-inflammatory than resveratrol, another plant phenolic compound found in grapes. More importantly, we found that the apigenin anti-inflammatory activity also reduces survival of stimulated monocytes in a model of lung inflammation [Doseff, unpubl. obs.]. Hence, the ability of apigenin and other flavonoids to modulate both innate immunity and monocyte life span may provide alternative therapies for inflammation.

\section{Monocyte and Macrophage Cell Fate}

Monocyte life span is determined by the integration of a complex network of survival and death signals [36]. In monocytes, a constitutively active cell death program determines their short life span. During inflammation or in the presence of differentiation factors, apoptosis is blocked, sustaining prolonged survival of monocytes. Interestingly, as inflammation resolves, the survival program is promptly halted and apoptosis reassumes. Thus, in monocytes, 'on and off' apoptotic switches control cell fate. Macrophages, however, have acquired mechanisms that inhibit the apoptotic program and activate survival pathways responsible for promoting a longer life span. In this section, we discuss general mechanisms that control monocyte and macrophage life span.

\section{Regulation of Monocyte Cell Death}

Programmed cell death or apoptosis is an evolutionarily conserved mechanism essential for normal development and defense against pathogens. Apoptosis is characterized by a group of biochemical and distinct morphological changes including nuclear fragmentation, cytoskeleton disruption, cell shrinkage and membrane blebbing, which then lead to the fragmentation of the dying cell into apoptotic bodies that are recognized and engulfed by macrophages [6]. In addition, apoptosis involves the activation of a well-conserved group of cysteine-proteases, the caspases. The caspases are constitutively expressed as inactive zymogens that become proteolytically active upon apoptosis (fig. 1). Functionally, the caspases are divided into 2 groups: inflammatory and apoptotic. Inflammatory caspases include caspases 1, 4, $5,11,12$, and 13 . The role of caspase- 1 in inflammation 
has been nicely documented. Caspase-1, identified originally in monocytes, is responsible for cleaving the inflammatory cytokines IL-1 $\beta$ and IL-18. Mice lacking caspase-1 show decreased inflammation when infected with bacteria or LPS. Inflammatory caspases assemble into multiprotein complexes termed 'inflammasomes', which are required for their activation. The expression and role of inflammasomes and inflammatory caspases have been studied in human monocytes, and findings suggest that they may have key roles in the pathogenesis of inflammatory diseases [37].

Based on their position in the apoptotic cascade, the caspases are classified as 'initiators' (caspases 1, 2, 8, 9 and 10) and 'executioners' (caspases 3, 6 and 7). Initiator caspases 1, 2, 4, 5 and 9 have a caspase recruitment domain located in the amino-terminal involved in protein-protein interaction. Caspases 8 and 10 contain death effector domains (DEDs) participating in protein-protein interactions. In contrast, the executioner caspases have a short amino-terminal or prodomain. Lack of crystal structures and evolutionary conservation limits our current understanding of how the amino-terminal domain contributes to the activation of the executioner caspases. Recently, we have shown that Hsp27 binds to the amino-terminal domain of caspase-3, inhibiting its second proteolytic cleavage [4]. Whether prodomains of other executioner caspases are able to mediate associations remains to be shown.

Apoptosis can be activated by 2 main pathways, the death receptor or 'extrinsic' and the mitochondria-mediated or 'intrinsic' pathways. The extrinsic pathway is triggered by the binding of ligands to the death cell surface receptors belonging to the TNF receptor family [38]. In monocytes, the extrinsic pathway is modulated by 2 major receptors belonging to the TNF family, the CD95 or Fas receptor (FasR) and the tumor necrosis (TNF)-related apoptosis-inducing receptors TRAIL-R1 and TRAIL-R2, also named DR4 and DR5, respectively. Activation of these receptors promotes the recruitment of the cytoplasmic adaptor proteins TRADD (TNFRSF1A-associated via death domain) and FADD (Fas-associated protein with death domain) which, in turn, activate caspase-8. Monocytes and macrophages treated with endogenous doses of TNF initiate an immune response that, in the case of monocytes, promotes prolonged survival. Thus, apoptosis through the extrinsic pathway is mainly mediated by the trimerization of FasR recruits the cytoplasmic adaptor protein FADD and TRAIL-R1/2 the cytoplasmic adaptor protein TNF receptor-associated protein (TRADD) forming the 'DISC complex' (fig. 1). FADD and TRADD contain DED able to recruit other DEDcontaining proteins [39]. For example, caspase- 8 and caspase-10 interaction with FADD and TRADD through DED-DED domains leads to their auto-proteolytic activation. Caspase- 8 can either directly or indirectly activate caspase-3. Caspase-10, which is closely related to caspase-8, was also shown to be recruited to the DISC complex, become activated and initiate signaling pathways resulting in apoptosis. The functional role of Fas in vivo was demonstrated as both FasR (lpr/lpr) and FasL (lpr/gid) knockout mice have increased numbers of inflammatory and resident subsets of monocytes $\left(\mathrm{CD} 14^{\mathrm{dim}} \mathrm{Gr}-1^{+} \mathrm{CX} 3 \mathrm{CR} 1^{\text {low }}\right.$ and $\mathrm{CD} 14^{\mathrm{dim}} \mathrm{Gr}-1^{-} \mathrm{CX} 3 \mathrm{CR} 1^{\text {high }}$, respectively), resulting in lymphadenopathy, splenomegaly, and in the accumulation of macrophages in the tissue including lung, liver and spleen [40]. Together, these findings highlight the importance of the Fas-mediated pathway in monocyte/macrophage cell fate.

In the intrinsic pathway, apoptotic stimuli including stress or DNA-damaging agents induce changes in mitochondria membrane permeabilization, allowing caspase- 9 activation. Upon stress, cytochrome $\mathrm{c}$ and Smac/ Diablo are released from the mitochondria. Cytochrome $c$ in the presence of ATP binds to the apoptotic protease activating factor 1 (Apaf-1) and caspase-9 forming the 'apoptosome' [36]. The apoptosome, first identified in monocytic leukemia cells, induces caspase-9 activation followed by cleavage of caspase-3. Smac/Diablo forms a complex with XIAP relieving caspase-3 from the inhibition of XIAP. Different molecules related to the Bcl-2 (Bcell lymphoma 2) family are involved in the regulation of mitochondrial permeabilization, providing a homeostatic balance that contributes to cell fate determination.

ROS, which are produced as a result of aerobic metabolism, mainly by mitochondria, are important regulators of apoptosis. Importantly, ROS produced as a result of respiratory burst did not trigger monocyte apoptosis. Reactive nitrogen species interplay with ROS in apoptosis and both have been widely involved in the extrinsic and intrinsic pathways [41]. Activation of Fas causes elevated levels of ROS and treatment with anti-oxidants such as $\mathrm{N}$-acetylcysteine and glutathione was shown to completely block Fas-mediated apoptosis in monocytes. Moreover, lowering ROS levels with vitamin C prevents Fas-dependent apoptosis in human monocytes [42]. In addition, activation of monocytes causes a massive generation of ROS, e.g. $\mathrm{O}_{2}^{-}, \mathrm{H}_{2} \mathrm{O}_{2},{ }^{1} \mathrm{O}_{2}$, and $\mathrm{OH}^{-}$, and chlorinated oxidants such as $\mathrm{HOCl}$ that accompanies increased production of cytokines, e.g. IL- $1 \beta$ and TNF $\alpha$. Numerous studies suggest that both the oxidative stress induced 
by ROS and increased production of pro-inflammatory cytokines contribute to inflammatory disease conditions. Thus, ROS produced by mitochondria seem to be central in the induction of apoptosis by regulating the extrinsic and intrinsic pathways.

Another important pro-apoptotic regulator of cell fate is the serine/threonine PKC $\delta$. The PKC family consists of multiple isoforms divided in 3 groups: classical, novel and atypical. The classical group of PKCs, have anti-apoptotic properties and important functions in pathogen activation, whereas the novel group has been described as pro-apoptotic [43]. The isoforms are differentially expressed in monocytes and macrophages, suggesting their possible role in monocyte differentiation and life span. Classical members of this family such as PKC $\alpha$ and $\beta$ increase during PMA stimulation and monocyte-/macrophage-induced differentiation resulting in a decrease in $\mathrm{PKC} \delta$ expression. Some of the PKC isoforms $(\mathrm{PKC} \delta,-\varepsilon$, and $-\zeta$ ) are cleaved by caspase- 3 , suggesting the existence of a feedback loop that may regulate the activity of these enzymes. We found that PKC $\delta$ associates and phosphorylates caspase-3, increasing its apoptotic activity [44].

Importantly and independent of the initiating stimuli, once apoptosis is initiated, executioner caspases become activated, cleaving proteins of diverse biological functions, ranging from transcription factors to kinases and phosphatases. In fact, some of the caspase substrates contribute to the formation of apoptotic bodies. Thus, the apoptotic process must be tightly regulated, suggesting the existence of multiple checkpoints that contribute to the timely activation of the caspase cascade. These checkpoints provide unique 'on' and 'off' switches to use as molecular targets to potentially control monocyte accumulation at sites of inflammation.

\section{Regulation of Monocyte Cell Survival}

Anti-apoptotic proteins work by inhibiting caspases or the activation of the apoptotic program. Phosphatidyl inositol 3-kinase (PI-3K)/Akt, ERK, Fas, TNF, heat shock proteins and anti-apoptotic molecules, among others, play key roles in determining monocyte life span by regulating gene transcription and inhibiting the apoptotic program.

PI-3Ks are central mediators of hematopoietic cell survival, producing 3 '-inositol phosphatidylinositol (PIP3) metabolites involved in a multitude of cellular events, such as mitogenic responses, differentiation, apoptosis, cytoskeletal organization, phagocytosis, oxidative burst,
TLR-mediated stimulation, and the assembly of the NADPH-dependent oxidase involved in microbial killing. In addition, PI-3K serves important roles in the activation of oxidase, including recruitment and localization of $\mathrm{p} 47^{\text {phox }}$ and $\mathrm{p} 40^{\text {phox }}$ to the membrane and activation of $\mathrm{p} 47^{\mathrm{phox}}$ and activation of Akt. The Akt/(protein kinase B) are serine/threonine kinases critical mediators of cell survival. Mice deficient in phosphatases that regulate Akt phosphorylation such as the SHIP (Src-homology 2-containing inositol 5' phosphatase) and PTEN (tensin homolog deleted on chromosome ten) suffer expansion of tissue macrophage populations. The Akt kinase has 3 isoforms, namely Akt1, Akt2, and Akt3, that share a highly conserved pleckstrin homology domain involved in membrane recruitment. Akt translocation to the cell membrane induces its phosphorylation by PDK1 and PDK2, resulting in the suppression of cellular apoptosis. Akt direct phosphorylation of caspase-9 inhibits the intrinsic apoptotic pathway, thereby blocking caspase- 3 activation [45]. In addition, survival signaling by PI-3K/Akt acts through modulation of $\mathrm{Bcl}-2$ expression through modulation of NF- $\kappa \mathrm{B}$ and by phosphorylation of the anti-apoptotic proteins Bad and XIAP. In the presence of survival factors, activation of Akt/PI-3K induces monocyte differentiation into tissue macrophages [46]. Akt activity also mediates prolonged monocyte survival stimulated by pro-inflammatory signals such as IL-1 $\beta$ and LPS [5]. This coordinated control by Akt helps maintaining mitochondrial membrane potential and prevents the cytochrome $\mathrm{c}$ release and other pro-apoptotic mediators. Maintaining mitochondria functions keeps cellular ATP production and normal metabolism, thereby preventing cells from dying by necrosis or autophagy. Through the activation of mTOR, Akt exerts control on the translation of nutrient transporters. Future studies in this area will provide a better understanding on how metabolism influences the homeostatic balance that determines cell death and survival.

In monocytes/macrophages the MAPK, including the extracellular signal-regulated kinase (ERK), the c-JNK, and p38, are hubs to multiple networks of survival [47]. ERK1/2 are activated in response to mitogens and growth factors, whereas JNK and $\mathrm{p} 38$ pathways are preferentially activated by stress-inducing agents. Stimulation of monocytes with LPS results in activation of the ERK, JNK and p38. ERK can phosphorylate a variety of cytoplasmic and nuclear substrates that control cellular growth, differentiation, survival and apoptosis. Some of the substrates, including phospholipases, cytoskeletal proteins and transcription factors such as Elk1 and NF-IL6, are responsible 
for inducing AP-1, a central regulator of cytokines and metalloprotease production. In addition, ERK promotes survival by increasing the expression of $\mathrm{Bcl}-2$ family proteins and by inhibiting expression of apoptotic proteins (IAP) and recently shown by phosphorylation of caspase-9 blocking its activation. Thus, survival switches operate at the level of caspases or upstream in the apoptotic cascade. Main upstream regulators are the Bcl-2 family proteins acting as pro- or anti-apoptotic proteins. This family includes the anti-apoptotic proteins Bcl-2, Bcl-xL, A1, Bcl-w, Mcl-1 and pro-apoptotic members. The pro-apoptotic family members are classified based on the number of conserved Bcl-2 homology $(\mathrm{BH})$ domains. One group composed of the Bax family includes $\mathrm{Bak}, \mathrm{Bax}$, and Box, which have three $\mathrm{BH} 3$ domains. The second group corresponds to the $\mathrm{BH} 3$-only domain proteins. Over-expression of Bcl-2 and Bcl-xL in monocytic cells increases the protection against apoptotic stimuli. This group includes Bad, Bid, Bik, Bmf, Bim, Hrk, Noxa and Puma. These proteins, through mechanisms still unclear, modulate release of cytochrome $\mathrm{c}$ and Smac/Diablo from the mitochondria to the cytoplasm (fig. 1). The presence of Bik, Bak, Bax, Bad and Bid has also been reported in the monocytic lineage. In addition, Mcl-1 is over-expressed in tissue macrophages isolated from rheumatoid arthritis patients whereas the silencing of Mcl-1 resulted in the induction of apoptosis. Dimerization of the Bcl-2 members results in pore formation allowing the release of apoptogenic factors to the cytoplasm. In monocytes, $\mathrm{Bax} / \mathrm{Bak}$ and Bim/Bcl-2 associate with the VDAC1 (Voltage Dependent Activation Channel 1) in the outer mitochondrial membrane contributing to activation of apoptosis by facilitating pore formation. Monocytic cells treated with TRAIL show caspase- 8 activation leading to the cleavage of Bid (truncated Bid, tBid). tBid translocation to the mitochondria promotes the oligomerization of Bax/Bak helping to induce cell death. Thus, Bcl-2 proteins act as 'guardians' of cell fate by regulating in pairs cellular survival.

\section{Apoptosis, Clearance and Dual Role of Monocytes/Macrophages in Resolution of Inflammation}

As professional phagocytes, monocytes/macrophages orchestrate the clearance of invading pathogens and apoptotic cells, playing fundamental roles in remodeling and immunity. Defective clearance of apoptotic bodies has been associated with autoimmunity and is crucial for the resolution of inflammation. Phagocytosis accelerates the resolution of inflammation by producing anti-inflammatory mediators such as TGF- $\beta$ and prostaglandin E2 and by down-regulation of pro-inflammatory cytokines [6]. Numerous specific surface receptors are involved in recognition of pathogens, but so far only a handful of receptors responsible for recognition of apoptotic cells have been described [48]. Notably, apoptotic cells were found to release lysophosphatidylcholine, due to the caspase-3-mediated activation of the calcium-independent phospholipase A2, inducing the 'eat-me' signal to attract monocytes/macrophages [49]. Whether these signals and receptors would be potential targets to eliminate unwanted cells should provide future areas for therapeutic intervention. The contribution of these receptors and the 'eat-me' signals in individual monocyte subpopulations will need to be investigated.

Resolution of acute inflammation also requires apoptosis of inflammatory cells, including monocytes/macrophages. Apoptotic caspases play a fundamental role by proteolytically dismantling cells by degrading proteins with diverse biological functions. We found that caspase- 3 activation is essential for $\mathrm{CD} 14^{+}$monocyte apoptosis inducing nuclear fragmentation [2]. Interestingly, selective depletion of $\mathrm{CD} 14^{+} \mathrm{CD} 16^{+}$upon glucocorticoid treatment may be due to the increased levels of expression of glucorcorticoid receptors in this subpopulation [50]. Changes in apoptosis between classical and non-classical monocytes have also been described in response to heat shock and exercise. Probably, these differences are in part due to the differential expression of heat-shock proteins and adaptive immunity described in monocyte subsets. We found that activation of caspase- 3 in $\mathrm{CD} 14^{+}$is tightly regulated by multiple checkpoints including proteolytic removal of its amino-terminal prodomain, phosphorylation by $\mathrm{PKC} \delta$, and its association with the Hsp27 and XIAP inhibitors. How stimulatory signals control these checkpoints will need further study. Whether the central hubs for the survival-apoptosis regulatory network are shared by different monocyte subpopulations will need further investigation.

\section{Conclusions and Future Directions}

Monocytes/macrophages are critical for in nate immunity, protecting us from self and non-self antigens. Homeostatic control of monocytes/macrophages is shared between pro- and anti-inflammatory molecules and is essential for the regulation of the immune system. In a con- 
stant tug-of-war, stimulatory and death signals determine monocyte/macrophage life span. As a more defined map of the signal transduction pathways emerges, the potential to recognize model networks of survival and apoptosis should provide additional therapeutic approaches to regulate accumulation of monocytes/macrophages at sites of inflammation.

\section{Acknowledgments}

Work in Dr. Doseff's lab is supported by grants NIH (R01 HL075040-01) and NSF-MCB (0542244). Dr. Eubank's work is supported by grant NCI K99 CA131552. We apologize to those of our colleagues who made important contributions but which were omitted due to space limitations.

\section{References}

1 Auffray C, Sieweke MH, Geissmann F: Blood monocytes: development, heterogeneity, and relationship with dendritic cells. Annu Rev Immunol 2009;27:669-692.

2 Fahy RJ, Doseff AI, Wewers MD: Spontaneous human monocyte apoptosis utilizes a caspase-3-dependent pathway that is blocked by endotoxin and is independent of caspase-1. J Immunol 1999;163:1755-1762.

3 Wiktor-Jedrzejczak W, Gordon S: Cytokine regulation of the macrophage (M phi) system studied using the colony stimulating factor1-deficient op/op mouse. Physiol Rev 1996; 76:927-947.

4 Voss OH, Batra S, Kolattukudy SJ, GonzalezMejia ME, Smith JB, Doseff AI: Binding of caspase-3 prodomain to heat shock protein 27 regulates monocyte apoptosis by inhibiting caspase-3 proteolytic activation. J Biol Chem 2007;282:25088-25099.

5 Goyal A, Wang Y, Graham MM, Doseff AI, Bhatt NY, Marsh CB: Monocyte survival factors induce AKT activation and suppress caspase-3. Am J Respir Cell Mol Biol 2002; 26:224-230.

6 Savill J, Fadok V: Corpse clearance defines the meaning of cell death. Nature 2000;407: 784-788.

7 Linker R, Gold R, Luhder F: Function of neurotrophic factors beyond the nervous system: inflammation and autoimmune demyelination. Crit Rev Immunol 2009;29:43-68.

$\checkmark 8$ Gonzalez-Mejia E, Doseff AI: Regulation of monocytes and macrophages cell fate. Front Biosci 2009;14:2413-2431.

>9 Grage-Griebenow E, Flad HD, Ernst M: Heterogeneity of human peripheral blood monocyte subsets. J Leukoc Biol 2001;69:1120.

10 Skrzeczynska-Moncznik J, Bzowska M, Loseke S, Grage-Griebenow E, Zembala M, Pryjma J: Peripheral blood CD14 $4^{\text {high }} \mathrm{CD} 16^{+}$ monocytes are main producers of IL-10. Scand J Immunol 2008;67:152-159.

-11 Ziegler-Heitbrock L: The CD14 ${ }^{+} \mathrm{CD} 16^{+}$blood monocytes: their role in infection and inflammation. J Leukoc Biol 2007;81:584-592.

12 Geissmann F, Jung S, Littman DR: Blood monocytes consist of two principal subsets with distinct migratory properties. Immunity 2003;19:71-82.
13 Faure S, Meyer L, Costagliola D, Vaneensberghe C, Genin E, Autran B, Delfraissy JF, McDermott DH, Murphy PM, Debre P, Theodorou I, Combadiere C: Rapid progression to AIDS in HIV+ individuals with a structural variant of the chemokine receptor CX3CR1. Science 2000;287:2274-2277.

14 Gordon S, Taylor PR: Monocyte and macrophage heterogeneity. Nat Rev Immunol 2005;5:953-964.

15 Ingersoll MA, Spanbroek R, Lottaz C, Gautier EL, Frankenberger M, Hoffmann R, Lang R, Haniffa M, Collin M, Tacke F, Habenicht AJ, Ziegler-Heitbrock L, Randolph GJ: Comparison of gene expression profiles between human and mouse monocyte subsets. Blood 2010;115:e10-e19.

16 Aschoff L: Das reticulo-emdotheliale system. Ergeb Inn Med Kinderheilkd 1924;26: $1-118$.

17 Stanley E, Lieschke GJ, Grail D, Metcalf D, Hodgson G, Gall JA, Maher DW, Cebon J, Sinickas V, Dunn AR: Granulocyte/macrophage colony-stimulating factor-deficient mice show no major perturbation of hematopoiesis but develop a characteristic pulmonary pathology. Proc Natl Acad Sci USA 1994;91:5592-5596.

18 Begg SK, Radley JM, Pollard JW, Chisholm OT, Stanley ER, Bertoncello I: Delayed hematopoietic development in osteopetrotic (op/op) mice. J Exp Med 1993;177:237-242.

19 van Furth R, Cohm ZA: The origin and kinetics of mononuclear phagocytes. J Exp Med 1968;128:415-435.

20 Taylor PR, Martinez-Pomares L, Stacey M, Lin HH, Brown GD, Gordon S: Macrophage receptors and immune recognition. Annu Rev Immunol 2005;23:901-944.

21 Kohro T, Tanaka T, Murakami T, Wada Y, Aburatani H, Hamakubo T, Kodama T: A comparison of differences in the gene expression profiles of phorbol 12-myristate 13-acetate differentiated THP-1 cells and human monocyte-derived macrophage. J Atheroscler Thromb 2004;11:88-97.

22 Balkwill F, Mantovani A: Inflammation and cancer: back to Virchow. Lancet 2001;357: 539-545.
23 Sica A, Bronte V: Altered macrophage differentiation and immune dysfunction in tumor development. J Clin Invest 2007;117:11551166.

24 Luo Y, Zhou H, Krueger J, Kaplan C, Lee SH, Dolman C, Markowitz D, Wu W, Liu C, Reisfeld RA, Xiang R: Targeting tumor-associated macrophages as a novel strategy against breast cancer. J Clin Invest 2006;116: 2132-2141.

25 Eubank TD, Roberts RD, Khan M, Curry JM, Nuovo GJ, Kuppusamy P, Marsh CB: Granulocyte macrophage colony-stimulating factor inhibits breast cancer growth and metastasis by invoking an anti-angiogenic program in tumor-educated macrophages. Cancer Res 2009;69:2133-2140.

26 Eubank TD, Roberts R, Galloway M, Wang Y, Cohn DE, Marsh CB: GM-CSF induces expression of soluble VEGF receptor-1 from human monocytes and inhibits angiogenesis in mice. Immunity 2004;21:831-842.

27 De Palma M, Murdoch C, Venneri MA, Naldini L, Lewis CE: Tie2-expressing monocytes: regulation of tumor angiogenesis and therapeutic implications. Trends Immunol 2007;28:519-524.

28 Waldburger JM, Suter T, Fontana A, AchaOrbea H, Reith W: Selective abrogation of major histocompatibility complex class II expression on extrahematopoietic cells in mice lacking promoter IV of the class II transactivator gene. J Exp Med 2001;194: 393-406.

29 Akira S, Takeda K, Kaisho T: Toll-like receptors: critical proteins linking innate and acquired immunity. Nat Immunol 2001;2:675680.

-30 Picard C, Puel A, Bonnet M, Ku CL, Bustamante J, Yang K, Soudais C, Dupuis S, Feinberg J, Fieschi C, Elbim C, Hitchcock R, Lammas D, Davies J, Al-Ghonaium A, AlRayes H, Al-Jumaah A, Al-Hajjar S, AlMoshen IZ, Frayha HH, Rucker R, Hawn TR, Aderem A, Tufenkeji H, Haraguchi S, Day NK, Good RA, Gugerot-Pocidalo MA, Ozinsky A, Casanova JL: Pyogenic bacterial infections in humans with IRAK-4 deficiency. Science 2003;299:2076-2079.

- 31 Akira S, Takeda K: Toll-like receptor signalling. Nat Immunol 2004;4:499-511. 
32 Reuter S, Lang D: Life span of monocytes and platelets: importance of interactions. Front Biosci 2009; 14:2432-2447.

- 33 Lekkou A, Karakantza M, Mouzaki A Kalfarentzos F, Gogos CA: Cytokine production and monocyte HLA-DR expression as predictors of outcome for patients with community-acquired severe infections. Clin Diagn Lab Immunol 2004;11:161-167.

34 Gaur U, Aggarwal BB: Regulation of proliferation, survival and apoptosis by members of the TNF superfamily. Biochem Pharmacol 2003;66:1403-1408.

35 Nicholas C, Batra S, Vargo MA, Voss OH, Gavrilin MA, Wewers MD, Guttdrige DC, Grotewold E, Doseff AI: Apigenin blocks lipopolysaccharide-induced lethality in vivo and pro-inflammatory cytokines expression by inactivating NF- $\kappa \mathrm{B}$ through the suppression of p65 phosphorylation. J Immunol 2007;179:7121-7127.

-36 Doseff AI: Apoptosis: the sculptor of development. Stem Cells Developm 2004;13:473483.

37 Fahy RJ, Exline MC, Gavrilin MA, Bhatt NY, Besecker BY, Sarkar A, Hollyfield JL, Duncan MD, Nagaraja HN, Knatz NL, Hall M, Wewers MD: Inflammasome mRNA expression in human monocytes during early septic shock. Am J Respir Crit Care Med 2008 177:983-988.
38 Ashkenazi A, Dixit VM: Death receptors: signaling and modulation. Science 1998;281: 1305-1308

39 Krammer PH: CD95 (APO-1/Fas)-mediated apoptosis: live and let die. Ad Immunol 1999; 71:163-209.

40 Nagata S: Fas ligand-induced apoptosis. Annu Rev Genet 1999;33:29-55.

-41 Parihar MS, Nazarewicz RR, Kincaid E, Bringold U, Ghafourifar P: Association of mitochondrial nitric oxide synthase activity with respiratory chain complex I. Biochem Biophys Res Commun 2008;366:23-28.

2 Perez-Cruz I, Carcamo JM, Golde DW: Vitamin $\mathrm{C}$ inhibits FAS-induced apoptosis in monocytes and U937 cells. Blood 2003;102: 336-343.

43 Malavez Y, Gonzalez-Mejia ME, Doseff AI: PKC $\delta$. Atlas Genet Cytogenet Oncol Haematol. March 2008 http://AtlasGeneticsOncology.org/Genes/PRKCDID42901ch3p21. html (accessed March, 2010).

44 Voss OH, Kim S, Wewers MD, Doseff AI: Regulation of monocyte apoptosis by the protein kinase $\mathrm{C}$ delta-dependent phosphorylation of caspase-3. J Biol Chem 2005;280: 17371-17379.
45 Cardone MH, Roy N, Stennicke HR, Salvesen GS, Franke TF, Stanbridge E, Frisch S, Reed JC: Regulation of cell death protease caspase- 9 by phosphorylation. Science 1998; 282:1318-1321.

46 Kelley TW, Graham MM, Doseff AI, Pomerantz RW, Lau SM, Ostrowski MC, Franke TF, Marsh CB: Macrophage colony-stimulating factor promotes cell survival through Akt/protein kinase B. J Biol Chem 1999;274: 26393-26398.

47 Chang L, Karin M: Mammalian MAP kinase signalling cascades. Nature 2001;410:37-40.

48 Erwig LP, Henson PM: Clearance of apoptotic cells by phagocytes. Cell Death Differ 2008;15:243-250.

-49 Lauber K, Bohn E, Krober SM, Xiao YJ, Blumenthal SG, Lindemann RK, Marini $P$, Wiedig C, Zobywalski A, Baksh S, Xu Y, Autenrieth IB, Schulze-Osthoff K, Belka C, Stuhler G, Wesselborg S: Apoptotic cells induce migration of phagocytes via caspase-3mediated release of a lipid attraction signal. Cell 2003;113:717-730.

50 Dayyani F, Belge KU, Frankenberger M, Mack M, Berki T, Ziegler-Heitbrock L: Mechanism of glucocorticoid-induced depletion of human $\mathrm{CD} 14^{+} \mathrm{CD} 16^{+}$monocytes. J Leukoc Biol 2003;74:33-39. 